\title{
FINITE-TIME STABILIZATION IN OPTIMAL TIME OF HOMOGENEOUS QUASILINEAR HYPERBOLIC SYSTEMS IN ONE DIMENSIONAL SPACE*
}

\author{
JeAn-Michel Coron ${ }^{1, * *}$ And HoAi-Minh NGuYen ${ }^{2}$
}

\begin{abstract}
We consider the finite-time stabilization of homogeneous quasilinear hyperbolic systems with one side controls and with nonlinear boundary condition at the other side. We present timeindependent feedbacks leading to the finite-time stabilization in any time larger than the optimal time for the null controllability of the linearized system if the initial condition is sufficiently small. One of the key technical points is to establish the local well-posedness of quasilinear hyperbolic systems with nonlinear, non-local boundary conditions.
\end{abstract}

Mathematics Subject Classification. 93D15, 35L50, 35L60.

Received February 27, 2020. Accepted September 22, 2020.

\section{INTRODUCTION AND STATEMENT OF THE MAIN RESULT}

Linear and nonlinear hyperbolic systems in one dimensional space are frequently used in modeling of many systems such as traffic flow, heat exchangers, and fluids in open channels. The stability and boundary stabilization of these hyperbolic systems have been studied intensively in the literature, see e.g. [2] and the references therein. In this paper, we investigate the finite-time stabilization in optimal time of the following homogeneous, quasilinear, hyperbolic system in one dimensional space

$$
\partial_{t} w(t, x)=\Sigma(x, w(t, x)) \partial_{x} w(t, x) \text { for }(t, x) \in[0,+\infty) \times(0,1) .
$$

Here $w=\left(w_{1}, \ldots, w_{n}\right)^{\top}:[0,+\infty) \times(0,1) \rightarrow \mathbb{R}^{n}, \Sigma(\cdot, \cdot)$ is an $(n \times n)$ real matrix-valued function defined in $[0,1] \times \mathbb{R}^{n}$. We assume that $\Sigma(\cdot, \cdot)$ has $m \geq 1$ distinct positive eigenvalues and $k=n-m \geq 1$ distinct negative eigenvalues. We assume that, maybe after a change of variables, $\Sigma(x, y)$ for $x \in[0,1]$ and $y \in \mathbb{R}^{n}$ is of the form

$$
\Sigma(x, y)=\operatorname{diag}\left(-\lambda_{1}(x, y), \ldots,-\lambda_{k}(x, y), \lambda_{k+1}(x, y), \ldots, \lambda_{k+m}(x, y)\right)
$$

* Dedicated to Enrique Zuazua, a friend and a great scientist, on the occasion of his 60th birthday.

Keywords and phrases: Stabilization, nonlinear 1-D hyperbolic systems, feedback laws.

1 Sorbonne Université, Université de Paris, CNRS, INRIA, Laboratoire Jacques-Louis Lions, équipe Cage, Paris, France.

2 Ecole Polytechnique Fédérale de Lausanne, EPFL, CAMA, Station 8, CH-1015 Lausanne, Switzerland.

** Corresponding author: coron@ann.jussieu.fr 
where

$$
-\lambda_{1}(x, y)<\cdots<-\lambda_{k}(x, y)<0<\lambda_{k+1}(x, y)<\cdots \lambda_{k+m}(x, y)
$$

Throughout the paper, we assume

$$
\lambda_{i} \text { is of class } C^{2} \text { with respect to } x \text { and } y \text { for } 1 \leq i \leq n=k+m \text {. }
$$

Denote

$$
w_{-}=\left(w_{1}, \ldots, w_{k}\right)^{\top} \text { and } w_{+}=\left(w_{k+1}, \ldots, w_{k+m}\right)^{\top} .
$$

The following types of boundary conditions and controls are considered. The boundary condition at $x=0$ is given by

$$
w_{-}(t, 0)=\mathrm{B}\left(w_{+}(t, 0)\right) \text { for } t \geq 0,
$$

for some

$$
\mathrm{B} \in\left(C^{2}\left(\mathbb{R}^{m}\right)\right)^{k} \text { with } \mathrm{B}(0)=0,
$$

and the boundary control at $x=1$ is

$$
w_{+}(t, 1)=\left(W_{k+1}, \ldots, W_{k+m}\right)^{\top}(t) \text { for } t \geq 0,
$$

where $W_{k+1}, \ldots, W_{k+m}$ are controls. In this work, we thus consider non-linear boundary condition at $x=0$.

Set

$$
\tau_{i}=\int_{0}^{1} \frac{1}{\lambda_{i}(x, 0)} \mathrm{d} x \text { for } 1 \leq i \leq n,
$$

and

$$
T_{\text {opt }}:=\left\{\begin{array}{cl}
\max \left\{\tau_{1}+\tau_{m+1}, \ldots, \tau_{k}+\tau_{m+k}, \tau_{k+1}\right\} & \text { if } m \geq k, \\
\max \left\{\tau_{k+1-m}+\tau_{k+1}, \tau_{k+2-m}+\tau_{k+2}, \ldots, \tau_{k}+\tau_{k+m}\right\} & \text { if } m<k .
\end{array}\right.
$$

The main result of this paper is the following result whose proof is given in the next section.

Theorem 1.1. Define

$$
\mathcal{B}:=\left\{B \in \mathbb{R}^{k \times m} ; \text { such that (1.10) holds for } 1 \leq i \leq \min \{m-1, k\}\right\},
$$

where

the $i \times i$ matrix formed from the last $i$ columns and the last $i$ rows of $B$ is invertible.

Assume that $B=\nabla \mathrm{B}(0) \in \mathcal{B}$. For any $T>T_{\text {opt }}$, there exist $\varepsilon>0$ and a time-independent feedback control for (1.1), (1.5), and (1.6) such that if the compatibility conditions $($ at $x=0)(1.13)$ and (1.14) hold for $w(0, \cdot)$,

$$
\left(\|w(0, \cdot)\|_{C^{1}([0,1])}<\varepsilon\right) \Rightarrow(w(T, \cdot)=0) .
$$


Remark 1.2. 1. The feedbacks constructed also lead to the well-posedness of the Cauchy problem for the closed loop system (see Lem. 2.3) and to the following property: for every $\eta>0$, there exists $\delta>0$ such that, if the compatibility conditions (at $x=0)(1.13)$ and $(1.14)$ hold for $w(0, \cdot)$,

$$
\left(\|w(0, \cdot)\|_{C^{1}([0,1])}<\delta\right) \Rightarrow\left(\|w(t, \cdot)\|_{C^{1}([0,1])}<\eta, \forall t \in[0, T]\right)
$$

see the proof of Lemma 2.3. Hence, by $(1.11)$ and $(1.12), 0 \in\left(C^{1}([0,1])\right)^{n}$ is stable for the closed-loop system and $0 \in\left(C^{1}([0,1])\right)^{n}$ is finite-time stable in time $T$. 2. The feedbacks constructed in this article use additional $4 m$ state-variables (dynamics extensions) to avoid imposing compatibility conditions at $x=1$. In particular, (1.11) and (1.12) are understood with these additional $4 m$ state-variables. 3. The null feedback law $\left(W_{k+1}, \ldots, W_{k+m}\right)^{\top}=0$ also leads to finite-time stability but in a time $T_{1}$ which can be larger than $T_{o p t}$ even in the linear case with $B \in \mathcal{B}$ if $m \geq 2$. Indeed for generic $B$ in $\in \mathcal{B}, T_{1}=\tau_{k}+\tau_{k+1}$ in the linear case $\mathrm{B}(\cdot)=B \cdot$. Moreover, if one denotes by $T_{1}(\varepsilon)$ the smallest value of $T$ such that (1.11) holds for the null feedback law, for every $B \in \mathcal{R}^{k \times m}$, for generic (for the $C^{2}$-topology) nonlinear $\mathrm{B} \in\left(C^{2}\left(\mathbb{R}^{m}\right)\right)^{k}$ such that $\mathrm{B}(0)=0$ and $\nabla \mathrm{B}(0)=B, T_{1}(\varepsilon) \rightarrow \tau_{k}+\tau_{k+1}$ as $\varepsilon \rightarrow 0^{+}$; it then suffices to point out that $\tau_{k}+\tau_{k+1} \geq T_{o p t}$ with equality if and only if $m=1$.

In what follows, we denote, for $x \in[0,1]$ and $y \in \mathbb{R}^{n}$,

$$
\Sigma_{-}(x, y)=\operatorname{diag}\left(-\lambda_{1}(x, y), \ldots,-\lambda_{k}(x, y)\right) \text { and } \Sigma_{+}(x, y)=\operatorname{diag}\left(\lambda_{k+1}(x, y), \ldots, \lambda_{n}(x, y)\right) .
$$

The compatibility conditions considered in Theorem 1.1 are:

$$
w_{-}(0,0)=\mathrm{B}\left(w_{+}(0,0)\right)
$$

and

$$
\Sigma_{-}(0, w(0,0)) \partial_{x} w_{-}(0,0)=\nabla \mathrm{B}\left(w_{+}(0,0)\right) \Sigma_{+}(0, w(0,0)) \partial_{x} w_{+}(0,0) .
$$

Null-controllability of hyperbolic systems with one side controls have been studied at least from the work of David Russell [16] even for inhomogeneous systems, i.e., instead of (1.1), one considers

$$
\partial_{t} w(t, x)=\Sigma(x, w(t . x)) \partial_{x} w(t, x)+C(x, w(t, x))
$$

for some $C \in\left(L^{\infty}\left([0,1] \times \mathbb{R}^{n}\right)\right)^{n \times n}$ with $C(x, 0)=0$. For linear systems, i.e., $\Sigma(x, \cdot)$ and $C(x, \cdot)$ are constant for $x \in[0,1]$ and $\mathrm{B}$ is linear $(\mathrm{B}(\cdot)=B \cdot$ with $B=\nabla \mathrm{B}(0))$, the null-controllability was established in ([16], Sect. 3) for the time $\tau_{k}+\tau_{k+1}$. Using backstepping approach, feedback controls leading to finite-time stabilization in the same time were then initiated by Jean-Michel Coron et al. in [7] for $m=k=1$ and later developed in [1,8] for the general case. The set $\mathcal{B}$ was introduced in [4] and the null-controllability for the linear systems with $B \in \mathcal{B}$ was established for $T>T_{\text {opt }}$ in [4,5] (see also [17] for the case $C$ diagonal) via the backstepping approach. A tutorial introduction of backstepping approach can be found in [11]. In the quasilinear case with $m \geq k$ and with the linear boundary condition at $x=0$, the null controllability for any time greater than $\tau_{k}+\tau_{k+1}$ was established for $m \geq k$ by Tatsien Li in [12], Theorem 3.2 (see also [13]).

This work is concerned about homogeneous quasilinear hyperbolic systems with controls on one side, and with nonlinear boundary conditions on the other side: (1.1), (1.5), and (1.6). The null-controllability is obtained in any time greater than $\tau_{k}+\tau_{k+1}$ using the zero controls if the initial data are sufficiently small. When the boundary condition is linear, the null-controllability was established by Long $\mathrm{Hu}$ [9] for $m \geq k$ at any time greater than $\max \left\{\tau_{k+1}, \tau_{k}+\tau_{m+1}\right\}$ if initial data are sufficiently small. In the linear case [4], for $B \in \mathcal{B}$, we obtained time-independent feedbacks for the null controllability at time $T_{o p t}$ and showed the optimality of $T_{o p t}$ 
if, moreover, (1.10) with $i=\min \{m, k\}$ holds (if this last condition does not hold, see Rem. 1.3). Related exact controllability results can be also found in $[4,9,10]$. In this work, for $\nabla \mathrm{B}(0) \in \mathcal{B}$, we present time-independent feedbacks leading to finite-time stabilization of (1.1), (1.5), and (1.6) in any time $T>T_{\text {opt }}$ provided that the initial data are sufficiently small. It is easy to see that $\mathcal{B}$ is an open subset of the set of (real) $k \times m$ matrices, and the Hausdorff dimension of its complement is $\min \{k, m-1\}$.

The feedbacks for (1.1), (1.5), and (1.6) are nonlinear and inspired from the ones in [4]. The construction is more complicated due to quasilinear nature of the system. We add auxiliary dynamics to fulfill the compatibility conditions at $x=1$ since $C^{1}$-solutions are considered. One of the key technical points is to establish the local well-posedness of quasilinear hyperbolic systems with nonlinear, non-local boundary conditions, which is interesting in itself. The Lyapunov functions associated with the feedbacks considered here are investigated in our forthcoming work [6].

Remark 1.3. Consider the linear case: $\mathrm{B}(\cdot)=B$. with $B \in \mathcal{B}, m \leq k$ and assume that (1.10) with $i=\min \{m, k\}=m$ does not hold. Then the optimal time for the null controllability of (1.1), (1.5), and (1.6) can be smaller than $T_{\text {opt }}$. Indeed, let

$$
J_{0}=\{1 \leq j \leq k-m+1 ; \text { the } j \text { th row of } B \text { is not in the space spanned by the last } m-1 \text { rows of } B\} .
$$

Set $\hat{\tau}_{0}=\tau_{j_{0}}+\tau_{k+1}$ where $j_{0}=\max _{j \in J_{0}} j$ if $J_{0}$ is not empty, and $\hat{\tau}_{0}=0$ otherwise. Define

$$
\hat{T}_{o p t}=\max \left\{\hat{\tau}_{0}, \tau_{k+2-m}+\tau_{k+2}, \ldots, \tau_{k}+\tau_{k+m}\right\} .
$$

Then the null controllability is attained for $\hat{T}_{o p t}$, which might be smaller than $T_{\text {opt }}$.

Indeed, we use the same control as in the proof of Proposition 1.6 from [4]. Consider first the case where $J_{0}$ is not empty. By the definition of $j_{0}$, it follows that, for $j_{0}+1 \leq j \leq k-m+1$,

$$
\left(B w_{+}\right)_{j}(t, 0) \text { is a linear combination of }\left(B w_{+}\right)_{k-m+2}(t, 0), \ldots,\left(B w_{+}\right)_{k}(t, 0) .
$$

One then derives from (1.16) that, for $j_{0}+1 \leq j \leq k-m+1$,

$$
(B w)_{j}(t, 0)=0 \text { for } t \geq \tau_{k+2}
$$

since $(B w)_{k}(t, 0)=0$ for $t \geq \tau_{k+m}, \ldots,(B w)_{k-m+2}(t, 0)=0$ for $t \geq \tau_{k+2}$. By the characteristic method, this implies, for $j_{0}+1 \leq j \leq k-m+1$,

$$
w_{j}(t, \cdot)=0 \text { for } t \geq \tau_{k+2}+\tau_{k+m-1} .
$$

On the other hand, from the proof of Proposition 1.6 from [4], we have

$$
\begin{aligned}
& w_{1}(t, \cdot)=\cdots=w_{j_{0}}(t, \cdot)=w_{k-m+2}(t, \cdot)=\cdots=w_{k+m}(t, \cdot)=0 \\
& \quad \text { for } t \geq \max \left\{\tau_{j_{0}}+\tau_{k+1}, \tau_{k+2-m}+\tau_{k+2}, \ldots, \tau_{k}+\tau_{k+m}\right\} .
\end{aligned}
$$

(One can also view this fact as a consequence of the proof of Proposition 1.6 from [4] applied to the system consisting of the first $j_{0}$ components and the last $2 m-1$ components of $w$ ). The null-controllability for $\hat{T}_{\text {opt }}$ now follows from (1.17) and (1.18) in this case.

We next consider the case where $J_{0}$ is empty. Similar to (1.17), we have, for $1 \leq j \leq k-m+1$,

$$
w_{j}(t, \cdot)=0 \text { for } t \geq \tau_{k+2}+\tau_{k-m+1}
$$


and similar to (1.18), we obtain

$$
w_{k-m+2}(t, \cdot)=\cdots=w_{k+m}(t, \cdot)=0 \text { for } t \geq \max \left\{\tau_{k+2-m}+\tau_{k+2}, \ldots, \tau_{k}+\tau_{k+m}\right\} .
$$

The null-controllability for $\hat{T}_{\text {opt }}$ in the case where $J_{0}$ is empty now follows from (1.19) and (1.20).

One can also show the optimality of $\hat{T}_{\text {opt }}$. When $J_{0}$ is not empty, this follows from the proof of Proposition 1.6 from [4] on the optimality part applied to the first $j_{0}$ components and the last $2 m-1$ components of $w$ after considering the initial data $w(t=0, \cdot)$ which satisfies $w_{j_{0}+1}(t=0, \cdot)=\cdots=w_{k-m+1}(t=0, \cdot)=0$. When $J_{0}$ is empty, this follows from the proof of Proposition 1.6 from [4] on the optimality part applied to the components $k-m+2, \ldots, k, k+2, \ldots k+m$.

Let us point out that, if $m \leq k$ and $\hat{T}_{o p t}<T_{o p t}$, Theorem 1.1 does not hold for $T_{o p t}$ replaced by $\hat{T}_{o p t}$ even if (1.10) with $i=\min \{m, k\}=m$ does not hold. In fact, even the local null controllability in any time $T<T_{\text {opt }}$ does not hold for generic (for the $C^{2}$-topology) nonlinear $\mathrm{B} \in\left(C^{2}\left(\mathbb{R}^{m}\right)\right)^{k}$ such that $\mathrm{B}(0)=0$ and $\nabla \mathrm{B}(0)=B$ where $B$ is any given element of in $\mathcal{B}$, whatever are $m \geq 1$ and $k \geq 1$.

The discussion on the case where $B \in \mathcal{B}, m \leq k$, and (1.10) with $i=\min \{m, k\}=m$ is not satisfied is motivated by exchanged messages with Guillaume Olive, who also informed us that with Long Hu he recently characterized the optimal time for the null controllability in the linear case and for every $B$. We are grateful for the discussions.

\section{Proof OF THE MAIN RESUlT}

This section containing two subsections is devoted to the proof of Theorem 1.1. In the first subsection, we establish the local well-posedness of quasilinear hyperbolic systems with nonlinear, non-local boundary conditions. This implies in particular the well-posedness for the feedback laws given in the proof of Theorem 1.1 associated with (1.1) and (1.5). The proof of Theorem 1.1 is given in the second subsection.

\subsection{Preliminaries}

The main result of this section is Lemma 2.3 on the well-posedness for quasi-linear hyperbolic systems related to (1.1) and (1.5). The assumptions made are guided by our feedback controls used in Theorem 1.1. We first consider the semilinear system, with $T>0$,

$$
\left\{\begin{array}{cl}
\partial_{t} u(t, x)=A(t, x) \partial_{x} u(t, x)+f(t, x, u(t, x)) & \text { in }[0, T] \times[0,1], \\
u_{-}(t, 0)=g\left(t, u_{+}(t, 0)\right) & \text { for } t \in[0, T], \\
u_{+}(t, 1)=h\left(t, u(t, \cdot), u_{0}\right) & \text { for } t \in[0, T], \\
u(0, \cdot)=u_{0}(\cdot) & \text { in }[0,1],
\end{array}\right.
$$

for

$$
A(t, x)=\operatorname{diag}\left(-\lambda_{1}(t, x), \ldots,-\lambda_{m}(t, x), \lambda_{m+1}(t, x), \ldots, \lambda_{m+k}(t, x)\right),
$$

where

$$
-\lambda_{1}(t, x)<\cdots<-\lambda_{m}(t, x)<0<\lambda_{m+1}(t, x)<\cdots<\lambda_{m+k}(t, x)
$$

and for $f:[0, T] \times[0,1] \times \mathbb{R}^{n} \rightarrow \mathbb{R}^{n}, g:[0, T] \times \mathbb{R}^{m} \rightarrow \mathbb{R}^{k}$, and $h:[0, T] \times\left(C^{1}([0,1])\right)^{n} \times\left(C^{1}([0,1])\right)^{n} \rightarrow \mathbb{R}^{m}$.

We have 
Lemma 2.1. Assume that $A$ is of class $C^{1}, f$, and $g$ are of class $C^{2}$,

$$
\begin{gathered}
h\left(t, \varphi, u_{0}\right)=h_{1}\left(t, u_{0}\right)+h_{2}\left(t, \varphi, u_{0}\right) \text { with } h_{1}\left(\cdot, u_{0}\right), h_{2} \text { are of class } C^{1}, \\
\lim _{\eta \rightarrow 0} \sup _{\left\|u_{0}\right\|_{C^{1}([0,1])} \leq \eta} \sup _{t>0}\left(\left|h_{1}\left(t, u_{0}\right)\right|+\left|\partial_{t} h_{1}\left(t, u_{0}\right)\right|\right)=0, \\
f(t, x, 0)=g(t, 0)=h_{2}(t, 0, \cdot)=0,
\end{gathered}
$$

and the following conditions hold, for some $C>0, a \in[0,1), 1 \leq p<+\infty$, and $\varepsilon_{0}>0$,

$$
\begin{aligned}
& \left|h\left(t, \hat{\varphi}, u_{0}\right)-h\left(t, \varphi, u_{0}\right)\right|+\left|\partial_{t} h\left(t, \hat{\varphi}, u_{0}\right)-\partial_{t} h\left(t, \varphi, u_{0}\right)\right| \\
& \quad \leq C\left(\left\|\left(\hat{\varphi}-\varphi, \hat{\varphi}^{\prime}-\varphi^{\prime}\right)\right\|_{C^{0}([0, a])}+\left\|\left(\hat{\varphi}-\varphi, \hat{\varphi}^{\prime}-\varphi^{\prime}\right)\right\|_{L^{p}(0,1)}\right),
\end{aligned}
$$

for all $\varphi, \hat{\varphi}, u_{0} \in\left(C^{1}([0,1])\right)^{n}$ with $\max \left\{\|\hat{\varphi}\|_{C^{1}([0,1])},\|\varphi\|_{C^{1}([0,1])},\left\|u_{0}\right\|_{C^{1}([0,1])}\right\}<\varepsilon_{0}$, and

$$
\begin{aligned}
&\left|\frac{\mathrm{d}}{\mathrm{d} t} h\left(s, \hat{v}(t, \cdot), u_{0}\right)\right|_{s=t}-\left.\frac{\mathrm{d}}{\mathrm{d} t} h\left(s, v(t, \cdot), u_{0}\right)\right|_{s=t} \mid \\
& \leq C\left(\left\|\left((\hat{v}-v)(t, \cdot), \partial_{t}(\hat{v}-v)(t, \cdot), \partial_{x}(\hat{v}-v)(t, \cdot)\right)\right\|_{C^{0}([0, a])}\right. \\
&\left.+\left\|\left((\hat{v}-v)(t, \cdot), \partial_{t}(\hat{v}-v)(t, \cdot), \partial_{x}(\hat{v}-v)(t, \cdot)\right)\right\|_{L^{p}(0,1)}\right)
\end{aligned}
$$

for all $\hat{v}, v \in\left(C^{1}([0, T] \times[0,1])\right)^{n}$ and $u_{0} \in\left(C^{1}([0,1])\right)^{n}$ with $\max \left\{\|\hat{v}\|_{C^{1}([0, T] \times[0,1])},\|v\|_{C^{1}([0, T] \times[0,1])}\right\}<\varepsilon_{0}$ and $\left\|u_{0}\right\|_{C^{1}([0,1])}<\varepsilon_{0}$. There exists $\varepsilon>0$ such that for $u_{0} \in\left(C^{1}([0,1])\right)^{n}$ satisfying the compatibility conditions (see (2.7)-(2.9)) with $\left\|u_{0}\right\|_{C^{1}([0,1])}<\varepsilon$, there is a unique solution $u \in\left(C^{1}([0, T] \times[0,1])\right)^{n}$ of (2.1).

We recall the following definition of compatibility conditions for $(2.1): u_{0} \in\left(C^{1}([0,1])\right)^{n}$ is said to satisfy the compatibility conditions if

$$
\begin{gathered}
u_{0,-}(0)=g\left(0, u_{0,+}(0)\right), \quad u_{0,+}(1)=h\left(0, u_{0}(\cdot), u_{0}\right), \\
\left(A(0,0) u_{0}^{\prime}(0)+f\left(0,0, u_{0}(0)\right)\right)_{-}=\partial_{t} g\left(0, u_{+}(0)\right)+\partial_{y_{+}} g\left(0, u_{+}(0)\right)\left(A(0,0) u_{0}^{\prime}(0)+f\left(0,0, u_{0}(0)\right)\right)_{+}, \\
\left(A(0,1) u_{0}^{\prime}(1)+f\left(0,1, u_{0}(1)\right)\right)_{+}=\partial_{t} h\left(0, u_{0}, u_{0}\right)+\partial_{y} h\left(0, u_{0}, u_{0}\right)\left(A(0,1) u_{0}^{\prime}(1)+f\left(0,1, u_{0}(1)\right)\right) .
\end{gathered}
$$

Here and in what follows, the partial derivatives are taken with respect to the notations $f(t, x, y), g\left(t, y_{+}\right)$, and $h\left(t, y, u_{0}\right)$.

Remark 2.2. The conditions $a<1$ and $p<+\infty$ are crucial in Lemma 2.1. 
Proof of Lemma 2.1. Set, for $u \in(C([0, T] \times[0,1]))^{n}$,

$$
\|u\|_{0}:=\max _{1 \leq i \leq n} \max _{(t, x) \in[0, T] \times[0,1]}\left|\mathrm{e}^{-L_{1} t-L_{2} x} u_{i}(t, x)\right|
$$

and, for $u \in\left(C^{1}([0, T] \times[0,1])\right)^{n}$,

$$
\|u\|_{1}:=\max \left\{\|u\|_{0},\left\|\partial_{t} u\right\|_{0},\left\|\partial_{x} u\right\|_{0}\right\}
$$

where $L_{1}$ and $L_{2}$ are two large, positive constants determined later.

Set

$$
\mathcal{O}_{\varepsilon}:=\left\{v \in\left(C^{1}([0, T] \times[0,1])\right)^{n} \text { with } v(0, \cdot)=u_{0},\right.
$$

$$
\left.\partial_{t} v(0,1)=A(0,1) u_{0}^{\prime}(1)+f\left(0,1, u_{0}(1)\right), \text { and }\|v\|_{1} \leq \varepsilon\right\}
$$

From now on, we assume implicitly that $\left\|u_{0}\right\|_{C^{1}([0,1])}$ is sufficiently small so that $\mathcal{O}_{\varepsilon}$ is not empty. For $v \in \mathcal{O}_{\varepsilon}$, let $u=\mathcal{F}(v)$ be the unique $C^{1}$-solution of the system

$$
\left\{\begin{array}{cl}
\partial_{t} u(t, x)=A(t, x) \partial_{x} u(t, x)+f(t, x, v(t, x)) & \text { in }[0, T] \times[0,1], \\
u_{-}(t, 0)=g\left(t, u_{+}(t, 0)\right) & \text { for } t \in[0, T], \\
u_{+}(t, 1)=h(t, v(t, \cdot)) & \text { for } t \in[0, T], \\
u(0, \cdot)=u_{0}(\cdot) & \text { in }[0,1] .
\end{array}\right.
$$

Here and in what follows, for notational ease, we ignore the dependence of $h$ on $u_{0}$ and denote $h(t, v(t, \cdot))$ instead of $h\left(t, v(t, \cdot), u_{0}\right)$. As in the proof of Lemma 3.2 from [4] by (2.4) and (2.5), and the fact that $f$ and $g$ are of class $C^{1}$, one can prove that $\mathcal{F}$ is contracting for $\|\cdot\|_{1}$-norm provided that $L_{2}$ is large and $L_{1}$ is much larger than $L_{2}$. The condition $0 \leq a<1$ and $1 \leq p<+\infty$ are essential for the existence of $L_{1}$ and $L_{2}{ }^{1}$ The existence and uniqueness of $u$ then follow. Moreover, there exist two constants $C_{1}, C_{2}>0$, independent of $u_{0}$ such that for $\left\|u_{0}\right\|_{C^{1}([0,1])} \leq C_{1} \varepsilon$ and $\|v\|_{1}<\varepsilon$, there exists a unique solution $u \in\left(C^{1}([0, T] \times[0,1])\right)^{n}$ and moreover,

$$
\|u\|_{C^{1}([0, T] \times[0,1])} \leq C_{2}\left(\left\|u_{0}\right\|_{C^{1}([0,1])}+\sup _{t>0}\left(\left|h_{1}\left(t, u_{0}\right)\right|+\left|\partial_{t} h_{1}\left(t, u_{0}\right)\right|\right)\right) .
$$

It follows from (2.3) that for $\varepsilon>0$ small, there exists a constant $0<C_{3}(\varepsilon)<\varepsilon$ small, independent of $u_{0}$, such that for $\left\|u_{0}\right\|_{C^{1}([0,1])} \leq C_{3}(\varepsilon)$ and $v \in \mathcal{O}_{\varepsilon}$, then

$$
\|\mathcal{F}(v)\|_{1} \leq \varepsilon \text { which implies in particular that } \mathcal{F}(v) \in \mathcal{O}_{\varepsilon} .
$$

We claim that, for $\left\|u_{0}\right\|_{C^{1}([0,1])} \leq C_{3}(\varepsilon)$ and $\varepsilon$ sufficiently small,

$$
\mathcal{F} \text { is a contraction mapping w.r.t. }\|\cdot\|_{1} \text { from } \mathcal{O}_{\varepsilon} \text { into } \mathcal{O}_{\varepsilon} \text {. }
$$

\footnotetext{
${ }^{1}$ We here clarify a misleading point in the definition of $\mathcal{F}(v)$ in [4], (3.10) in the proof of Lemma 3.2 from [4]. Concerning this definition, in the RHS of [4], $(3.8), v_{j+k}(t, 0)$ must be understood as $(\mathcal{F}(v))_{j+k}(t, 0)$ and $(\mathcal{F}(v))_{j+k}(t, 0)$ is then determined by the RHS of [4], (3.6) or (3.7) as mentioned there. Related to this point, $V_{j}(t, 0)$ for $k+1 \leq j \leq k+m$ in [4], (3.14) and in the inequality just below must be replaced by $(\mathcal{F}(v)-\mathcal{F}(\hat{v}))_{j}$. The rest of the proof is unchanged.
} 
Indeed, fix $\lambda \in(0,1)$. As in the proof of Lemma 3.2 from [4], applying the characteristic method, and using (2.4) and (2.5), and the fact $f$ and $g$ are of class $C^{1}$, we obtain

$$
\|\mathcal{F}(\hat{v})-\mathcal{F}(v)\|_{0} \leq \lambda\|\hat{v}-v\|_{1}
$$

if $L_{2}$ is large and $L_{1}$ is much larger than $L_{2}$. Set $U(t, x)=\partial_{t} u(t, x)$ for $(t, x) \in[0, T] \times[0,1]$. We have

$$
\left\{\begin{array}{c}
\partial_{t} U(t, x)=A(t, x) \partial_{x} U(t, x)+\partial_{t} A(t, x) A(t, x)^{-1} U(t, x)+f_{1}(t, x, v) \text { in }[0, T] \times[0,1], \\
U_{-}(t, 0)=g_{1}(t) \text { for } t \in[0, T] \\
U_{+}(t, 1)=h_{1}(t) \text { for } t \in[0, T] \\
U(0, x)=A(0, x) u_{0}^{\prime}(x)+f\left(0, x, u_{0}(x)\right) \text { in }[0,1],
\end{array}\right.
$$

where

$$
\begin{gathered}
f_{1}(t, x, v)=-\partial_{t} A(t, x) A^{-1}(t, x) f(t, x, v(t, x))+\partial_{t} f(t, x, v(t, x))+\partial_{y} f(t, x, v(t, x)) \partial_{t} v(t, x) . \\
g_{1}(t)=\partial_{t} g\left(t, u_{+}(t, 0)\right)+\partial_{y_{+}} g\left(t, u_{+}(t, 0)\right) U_{+}(t, 0), \\
h_{1}(t)=\partial_{t} h(t, v(t, \cdot))+\partial_{y} h(t, v(t, \cdot)) \partial_{t} v(t, \cdot) .
\end{gathered}
$$

Note that, with $\hat{u}=\mathcal{F}(\hat{v})$ and $\hat{U}=\partial_{t} \hat{u}$,

$$
\begin{aligned}
& \left|\partial_{t} g\left(t, \hat{u}_{+}(t, 0)\right)+\partial_{y_{+}} g\left(t, \hat{u}_{+}(t, 0)\right) \hat{U}_{+}(t, 0)-\partial_{t} g\left(t, u_{+}(t, 0)\right)-\partial_{y_{+}} g\left(t, u_{+}(t, 0)\right) U_{+}(t, 0)\right| \\
& \quad \stackrel{g \in C^{2}}{\leq} C\left(\left|\hat{u}_{+}(t, 0)-u_{+}(t, 0)\right|+\left|\hat{U}_{+}(t, 0)-U_{+}(t, 0)\right|\right)
\end{aligned}
$$

and

$$
\left|f_{1}(t, x, \hat{v})-f_{1}(t, x, v)\right| \stackrel{f \in C^{2}}{\leq} C\left(|\hat{v}(t, x)-v(t, x)|+\left|\partial_{t} \hat{v}(t, x)-\partial_{t} v(t, x)\right|\right)
$$

and by (2.5) and (2.6),

$$
\begin{aligned}
\left|\partial_{t} h(t, v(t, \cdot))+\partial_{y} h(t, v(t, \cdot)) \partial_{t} v(t, \cdot)-\partial_{t} h(t, \hat{v}(t, \cdot))-\partial_{y} h(t, \hat{v}(t, \cdot)) \partial_{t} \hat{v}(t, \cdot)\right| \\
\leq C\left(\left\|\left((\hat{v}-v)(t, \cdot), \partial_{t}(\hat{v}-v)(t, \cdot), \partial_{x}(\hat{v}-v)(t, \cdot)\right)\right\|_{C^{0}([0, a])}\right. \\
\left.\quad+\left\|\left((\hat{v}-v)(t, \cdot), \partial_{t}(\hat{v}-v)(t, \cdot), \partial_{x}(\hat{v}-v)(t, \cdot)\right)\right\|_{L^{p}(0,1)}\right)
\end{aligned}
$$


if $\max \left\{\|u\|_{1},\|v\|_{1},\|\hat{u}\|_{1},\|\hat{v}\|_{1}\right\}<\varepsilon_{0}$. Again, as in the proof of Lemma 3.2 from [4], applying the characteristic method and using (2.5) and (2.6), we also have, by (2.15),

$$
\left\|\partial_{t} \mathcal{F}(\hat{v})-\partial_{t} \mathcal{F}(v)\right\|_{0} \leq \lambda\|\hat{v}-v\|_{1}
$$

Since

$$
\partial_{t}(\hat{u}-u)(t, x)=A(t, x) \partial_{x}(\hat{u}-u)(t, x)+f(t, x, \hat{v}(t, x))-f(t, x, v(t, x))
$$

and

$$
|f(t, x, \hat{v}(t, x))-f(t, x, v(t, x))| \leq C|\hat{v}(t, x)-v(t, x)|,
$$

it follows from (2.15) and (2.17) that

$$
\|\mathcal{F}(\hat{v})-\mathcal{F}(v)\|_{1} \leq C \lambda\|\hat{v}-v\|_{1} .
$$

Claim (2.14) is proved.

The existence and uniqueness of solutions of $(2.1)$ in $\left(C^{1}([0, T] \times[0,1])\right)^{n}$ now follow for $u_{0}$ satisfying $\left\|u_{0}\right\|_{C^{1}([0,1])} \leq C_{3}(\varepsilon)$. The proof is complete.

We next establish the key result of this section. To this end, we first set, for $\tau>0$,

$$
\begin{aligned}
\hat{\mathcal{D}}_{\tau}:= & \left\{\left(\Xi, \varphi, w_{0}\right) \in\left(C^{1}([0,+\infty) \times[0,1])\right)^{n} \times\left(C^{1}([0,1])\right)^{n} \times\left(C^{1}([0,1])\right)^{n}\right. \\
& \left.\max \left\{\|\Xi\|_{C^{1}([0,+\infty) \times[0,1])},\|\varphi\|_{C^{1}([0,1])},\left\|w_{0}\right\|_{C^{1}([0,1])}\right\}<\tau\right\}
\end{aligned}
$$

and, for $T>0$,

$$
\mathcal{D}_{\tau}:=\left\{\left(\Xi, w_{0}\right) ;\left(\Xi, 0, w_{0}\right) \in \hat{\mathcal{D}}_{\tau}, \Xi(0, \cdot)=w_{0}(\cdot), \Xi(t, \cdot)=0 \text { for } t>T,\right.
$$

and the compatibility conditions at $x=0$ hold for the system $(2.28)\}$.

The set $\mathcal{D}_{\tau}$ also depends on $T$ but we ignore this dependence explicitly for notational ease.

We have

Lemma 2.3. Let $T>0, f:[0,+\infty) \times[0,1] \times \mathbb{R}^{n} \rightarrow \mathbb{R}^{n}$ be of class $C^{2}$ such that $f(t, x, 0)=0$ for $(t, x) \in$ $[0,+\infty) \times[0,1]$. Assume that $B=\nabla \mathrm{B}(0) \in \mathcal{B}, \Sigma$ is of class $C^{2}$, and there exist $\tau>0$ and

$$
H:[0,+\infty) \times \hat{\mathcal{D}}_{\tau} \rightarrow \mathbb{R}^{m}
$$

such that $H$ is continuously differentiable w.r.t. $(t, \Xi, \varphi)$, and for some $C>0,1 \leq p<+\infty$, and $a \in[0,1)$, the following conditions hold, for $\left(\Xi, \varphi, w_{0}\right),\left(\hat{\Xi}, \hat{\varphi}, w_{0}\right) \in \hat{\mathcal{D}}_{\tau}$ with $\left(\Xi, w_{0}\right),\left(\hat{\Xi}, w_{0}\right) \in \mathcal{D}_{\tau}$,

$$
\begin{gathered}
H\left(t, \Xi, \varphi, w_{0}\right)=H_{1}\left(t, w_{0}\right)+H_{2}\left(t, \Xi, \varphi, w_{0}\right) \text { with } H_{1}\left(\cdot, w_{0}\right), H_{2} \text { are of class } C^{1}, \\
\lim _{\eta \rightarrow 0} \sup _{\left\|u_{0}\right\|_{C^{1}([0,1])} \leq \eta} \sup _{t>0}\left(\left|H_{1}\left(t, u_{0}\right)\right|+\left|\partial_{t} H_{1}\left(t, u_{0}\right)\right|\right)=0,
\end{gathered}
$$




$$
\begin{gathered}
\left|H_{2}\left(t, \Xi, \varphi, w_{0}\right)\right| \leq C\left(\left\|\left(\varphi, \varphi^{\prime}\right)\right\|_{C^{0}([0, a])}+\left\|\left(\varphi, \varphi^{\prime}\right)\right\|_{L^{p}(0,1)}\right), \\
\left|H\left(t, \hat{\Xi}, \hat{\varphi}, w_{0}\right)-H\left(t, \Xi, \varphi, w_{0}\right)\right|+\left|\partial_{t} H\left(t, \hat{\Xi}, \hat{\varphi}, w_{0}\right)-\partial_{t} H\left(t, \Xi, \varphi, w_{0}\right)\right| \\
\leq C\left(\|\hat{\Xi}-\Xi\|_{C^{0}([0,+\infty) \times[0,1])}\|\hat{\varphi}\|_{C^{1}([0,1])}+\|\hat{\varphi}-\varphi\|_{C^{0}([0, a])}+\|\hat{\varphi}-\varphi\|_{L^{p}([0,1])}\right), \\
\left|\left\langle\partial_{\varphi} H\left(t, \Xi, \varphi, w_{0}\right), d \varphi\right\rangle\right| \leq C\left(\|d \varphi\|_{C^{0}([0, a])}+\|d \varphi\|_{L^{p}(0,1)}\right) \quad \forall d \varphi \in\left(C^{1}([0,1])\right)^{n}, \\
\left|\frac{\mathrm{d}}{\mathrm{d} t} H\left(s, \Xi(t+\cdot, \cdot), \varphi, w_{0}\right)\right|_{s=t} \mid \leq C\left(\|\Xi(t+\cdot, \cdot)\|_{C^{1}([0,+\infty) \times[0,1])}+\|\varphi\|_{C^{1}([0,1])}\right)\|\varphi\|_{C^{1}([0,1])}, \\
\left|H_{1}\left(t^{\prime}, w_{0}\right)-H_{1}\left(t, w_{0}\right)\right| \leq \rho_{1}\left(c \eta, w_{0}\right),
\end{gathered}
$$

and, for $\eta>0$ and for $0 \leq\left|t^{\prime}-t\right| \leq \eta$, for $d \varphi, d \hat{\varphi} \in\left(C^{1}([0,1])\right)^{n}$,

$$
\begin{aligned}
& \left|\frac{\mathrm{d}}{\mathrm{d} s} H_{2}\left(s, \Xi(s+\cdot, \cdot), \hat{\varphi}, w_{0}\right)\right|_{s=t^{\prime}}-\left.\frac{\mathrm{d}}{\mathrm{d} s} H_{2}\left(s, \Xi(s+\cdot, \cdot), \varphi, w_{0}\right)\right|_{s=t} \mid \\
& \quad+\left|\left\langle\partial_{\varphi} H_{2}\left(t^{\prime}, \Xi, \hat{\varphi}, w_{0}\right), d \hat{\varphi}\right\rangle-\left\langle\partial_{\varphi} H_{2}\left(t, \Xi, \varphi, w_{0}\right), d \varphi\right\rangle\right| \\
& \quad \leq C\left(\rho_{1}\left(c \eta, w_{0}\right)+\rho_{2}(c \eta, \varphi, \hat{\varphi}, d \varphi, d \hat{\varphi})\right),
\end{aligned}
$$

for some constant $c>0$ and some function $\rho_{1}$ such that

$$
\lim _{\eta \rightarrow 0} \rho_{1}\left(\eta, w_{0}\right)=0
$$

where

$$
\begin{gathered}
\rho_{2}(\eta, \varphi, \hat{\varphi}, d \varphi, d \hat{\varphi})=\left\|\sup _{\substack{y \\
|y-x| \leq \eta}}\{|\varphi(y)-\hat{\varphi}(x)|+|d \varphi(y)-d \hat{\varphi}(x)|\}\right\|_{L^{p}(0,1)} \\
+\left\|\sup _{\substack{y \\
|y-x| \leq \eta}}\{|\varphi(y)-\varphi(x)|+|d \varphi(y)-d \varphi(x)|\}\right\|_{C([0, a])} .
\end{gathered}
$$

Assume also that for all $\left(\Xi, w_{0}\right) \in \mathcal{D}_{\tau}$, the system

$$
\left\{\begin{array}{cl}
\partial_{t} \widetilde{w}(t, x)=\Sigma(x, \Xi(t, x)) \partial_{x} \widetilde{w}(t, x)+f(t, x, \widetilde{w}(t, x)) & \text { in }[0,+\infty) \times[0,1], \\
\widetilde{w}_{-}(t, 0)=\mathrm{B}\left(\widetilde{w}_{+}(t, 0)\right) & \text { for } t \in[0,+\infty), \\
\widetilde{w}_{+}(t, 1)=H\left(t, \Xi(t+\cdot, \cdot), \widetilde{w}(t, \cdot), w_{0}\right) & \text { for } t \in[0,+\infty), \\
\widetilde{w}(0, \cdot)=w_{0}(\cdot) & \text { in }[0,1]
\end{array}\right.
$$

has a unique $C^{1}$-solution satisfying $\widetilde{w}(t, \cdot)=0$ for $t>T$. There exists $\varepsilon>0$ such that if $\|w(0, \cdot)\|_{C^{1}([0,1])}<\varepsilon$ and $w(0, \cdot)$ satisfies the compatibility conditions at $x=0$, then there is a unique solution $w \in\left(C^{1}([0, T] \times[0,1])\right)^{n}$ 
of (1.1) and (1.5) with

$$
w(t, 1)=H\left(t, w(t+\cdot, \cdot), w(t, \cdot), w_{0}\right) \text { for } t \in[0,+\infty)
$$

Moreover,

$$
\|w\|_{C^{1}([0,+\infty) \times[0,1])} \leq C\left(\left\|w_{0}\right\|_{C^{1}([0,1])}+\sup _{\left\|u_{0}\right\|_{C^{1}([0,1])} \leq \eta} \sup _{t>0}\left(\left|H_{1}\left(t, u_{0}\right)\right|+\left|\partial_{t} H_{1}\left(t, u_{0}\right)\right|\right)\right),
$$

for some positive constant independent of $w_{0}$ and $\varepsilon$.

In Lemma 2.3 and what follows, $\Xi(t+\cdot, \cdot)$ denotes the function $(s, x) \mapsto \Xi(t+s, x)$ and $w(t+\cdot, \cdot)$ denotes the function $(s, x) \mapsto w(t+s, x)$.

The compatibility conditions at $x=0$ considered in the context of Lemma 2.3 are

$$
w_{0,-}(0)=B\left(w_{0,+}(0)\right),
$$

and

$$
\left(\Sigma(0, \Xi(0,0)) \partial_{x} w(0,0)+f(0,0, w(0,0))\right)_{-}=\nabla B\left(w_{+}(0,0)\right)\left(\Sigma(0, \Xi(0,0)) \partial_{x} w(0,0)+f(0,0, w(0,0))\right)_{+} .
$$

The compatibility at $x=1$ of (2.28) is a part of the assumption of Lemma 2.3.

Before giving the proof of Lemma 2.3, let us discuss the motivation for the assumptions made. To this end, we present one of its applications used in the proof of Theorem 1.1. Consider the setting given in Theorem 1.1; $f=0$ in Lemma 2.3 then. For $\Xi \in\left(C^{1}([0,+\infty) \times[0,1])\right)^{n}$, define the flows

$$
\frac{\mathrm{d}}{\mathrm{d} t} x_{j}^{\Xi}(t, s, \xi)=\lambda_{j}\left(x_{j}^{\Xi}(t, s, \xi), \Xi\left(t, x_{j}^{\Xi}(t, s, \xi)\right)\right) \quad \text { and } \quad x_{j}^{\Xi}(s, s, \xi)=\xi \text { for } 1 \leq j \leq k,
$$

and

$$
\frac{\mathrm{d}}{\mathrm{d} t} x_{j}^{\Xi}(t, s, \xi)=-\lambda_{j}\left(x_{j}^{\Xi}(t, s, \xi), \Xi\left(t, x_{j}^{\Xi}(t, s, \xi)\right)\right) \quad \text { and } \quad x_{j}^{\Xi}(s, s, \xi)=\xi \text { for } k+1 \leq j \leq k+m \text {. }
$$

Here and in what follows, we only consider the flows with $x_{j}^{\Xi}(t, s, \xi) \in[0,1]$ so that $\Xi$ is well-defined. Assume that $m>k$. Since $\nabla \mathrm{B}(0) \in \mathcal{B}$, by the implicit theorem and the Gaussian elimination method, there exist $M_{k}: U_{k} \rightarrow \mathbb{R}, \ldots, M_{1}: U_{1} \rightarrow \mathbb{R}$ of class $C^{2}$ for some neighborhoods $U_{k}$ of $0 \in \mathbb{R}^{m-1}, \ldots, U_{1}$ of $0 \in \mathbb{R}^{m-k}$ such that, for $y_{+}=\left(y_{k+1}, \ldots, y_{k+m}\right)^{\top} \in \mathbb{R}^{m}$ with sufficiently small norm, the following facts hold

$$
\begin{gathered}
\left(\mathrm{B}\left(y_{+}\right)\right)_{k}=0 \text { if } y_{k+m}=M_{k}\left(y_{k+1}, \ldots, y_{k+m-1}\right), \\
\left(\mathrm{B}\left(y_{+}\right)\right)_{k}=\left(\mathrm{B}\left(y_{+}\right)\right)_{k-1}=0 \text { if } y_{k+m}=M_{k}\left(y_{k+1}, \ldots, y_{k+m-1}\right), y_{k+m-1}=M_{k-1}\left(y_{k+1}, \ldots, y_{k+m-2}\right), \\
\mathrm{B}\left(y_{+}\right)=0 \text { if } y_{k+m}=M_{k}\left(y_{k+1}, \ldots, y_{k+m-1}\right), \ldots, y_{m+1}=M_{1}\left(y_{k+1}, \ldots, y_{m}\right) .
\end{gathered}
$$


For $T>T_{o p t}$, set $\delta=T-T_{\text {opt }}$. Consider $\zeta_{j}$ and $\eta_{j}$ of class $C^{1}$ for $k+1 \leq j \leq k+m$ and for $t \geq 0$ satisfying

$$
\zeta_{j}(0)=w_{0, j}(1), \quad \zeta_{j}(t)=0 \text { for } t \geq \delta / 2, \quad \eta_{j}(0)=1, \quad \eta_{j}(t)=0 \text { for } t \geq \delta / 2,
$$

and

$$
\zeta_{j}^{\prime}(0)=\lambda_{j}\left(1, w_{0}(1)\right) w_{0, j}^{\prime}(1), \quad \eta_{j}^{\prime}(0)=0
$$

For $\left(\Xi, \varphi, w_{0}\right) \in \mathcal{D}_{\tau}$ with small $\tau$, set

$$
\begin{aligned}
& \left(H\left(t, \Xi, \varphi, w_{0}\right)\right)_{m}=\zeta_{k+m}(t) \\
& \quad+\left(1-\eta_{k+m}(t)\right) M_{k}\left(\varphi_{k+1}\left(x_{k+1}^{\Xi}\left(t, t+t_{m+k}^{\Xi}, 0\right)\right), \ldots, \varphi_{k+m-1}\left(x_{k+m-1}^{\Xi}\left(t, t+t_{m+k}^{\Xi}, 0\right)\right)\right), \\
& \left(H\left(t, \Xi, \varphi, w_{0}\right)\right)_{m-1}=\zeta_{k+m-1}(t) \\
& \quad+\left(1-\eta_{k+m-1}(t)\right) M_{k-1}\left(\varphi_{k+1}\left(x_{k+1}^{\Xi}\left(t, t+t_{m+k-1}^{\Xi}, 0\right)\right), \ldots, \varphi_{k+m-2}\left(x_{k+m-2}^{\Xi}\left(t, t+t_{m+k-1}^{\Xi}, 0\right)\right)\right), \\
& \left(H\left(t, \Xi, \varphi, w_{0}\right)\right)_{m+1-k}=\zeta_{m+1}(t) \\
& \quad+\left(1-\eta_{m+1}(t)\right) M_{1}\left(\varphi_{k+1}\left(x_{k+1}^{\Xi}\left(t, t+t_{m+1}^{\Xi}, 0\right)\right), \ldots, \varphi_{m}\left(x_{m}^{\Xi}\left(t, t+t_{m+1}^{\Xi}, 0\right)\right)\right),
\end{aligned}
$$

and

$$
\left(H\left(t, \Xi, \varphi, w_{0}\right)\right)_{j}=\zeta_{k+j}(t) \text { for } 1 \leq j \leq m-k,
$$

where $t_{j}^{\Xi}=t_{j}^{\Xi}(t)$ are defined by

$$
x_{m+k}^{\Xi}\left(t+t_{m+k}^{\Xi}, t, 1\right)=0, \ldots, x_{1+k}^{\Xi}\left(t+t_{1+k}^{\Xi}, t, 1\right)=0 \text { for } k+1 \leq j \leq k+m .
$$

We now show that $H$ satisfies the assumptions given in Lemma 2.3 if $\left\|w_{0}\right\|_{C^{1}([0,1])} \leq \varepsilon$ and $\varepsilon$ is sufficiently small ( $\tau$ is sufficiently small as well). We first note that the solutions of the system (2.28) are 0 for $t>T$ if $\|\Xi\|_{C^{1}([0,+\infty) \times[0,1])}$ is sufficiently small. The proof of this fact follows from the choice of $M_{j}$ (see the proof of (2.68)-(2.69) in the proof of Thm. 1.1). One can easily check that (2.18), (2.20), (2.22), (2.23), and (2.24) hold. Assertion (2.19) will be a consequence of our construction Lemma $3.2 \eta_{j}$ and $\zeta_{j}$ given later. We are next concerned about (2.21). It suffices to prove that

$$
\begin{aligned}
& \left|H\left(t, \Xi, \varphi, w_{0}\right)-H\left(t, \hat{\Xi}, \varphi, w_{0}\right)\right|+\left|\partial_{t} H\left(t, \Xi, \varphi, w_{0}\right)-\partial_{t} H\left(t, \hat{\Xi}, \varphi, w_{0}\right)\right| \\
& \quad \leq C\|\hat{\Xi}-\Xi\|_{C^{0}([0,+\infty) \times[0,1])}\|\varphi\|_{C^{1}([0,1])} .
\end{aligned}
$$

We claim that, for $1 \leq j \leq k+m$.

$$
\left|x_{j}^{\hat{\Xi}}(t, s, \xi)-x_{j}^{\Xi}(t, s, \xi)\right| \leq C\|\hat{\Xi}-\Xi\|_{C^{0}([0,+\infty) \times[0,1])}
$$


for $(t, s, \xi)$ so that both flows are well-defined. We only consider the case $k+1 \leq j \leq k+m$, the other cases can be proved similarly. We have

$$
\left|x_{j}^{\hat{\Xi}}(t, s, \xi)-x_{j}^{\Xi}(t, s, \xi)\right| \leq C\|\hat{\Xi}-\Xi\|_{C^{0}([0,+\infty) \times[0,1])}+C \int_{\min \{t, s\}}^{\max \{t, s\}}\left|x_{j}^{\hat{\Xi}}\left(s^{\prime}, s, \xi\right)-x_{j}^{\Xi}\left(s^{\prime}, s, \xi\right)\right| \mathrm{d} s^{\prime}
$$

and (2.38) follows.

Since, for $k+1 \leq j \leq k+m$,

$$
\int_{t}^{t+t_{j}^{\hat{\Xi}}} \lambda_{j}\left(x_{j}^{\hat{\Xi}}(s, t, 1), \hat{\Xi}\left(t, x_{j}^{\hat{\Xi}}(s, t, 1)\right)\right) \mathrm{d} s=1=\int_{t}^{t+t_{j}^{\Xi}} \lambda_{j}\left(x_{j}^{\Xi}(s, t, 1), \Xi\left(t, x_{j}^{\Xi}(s, t, 1)\right)\right) \mathrm{d} s,
$$

it follows from (1.3) and (2.38) that

$$
\begin{aligned}
\left|t_{j}^{\hat{\Xi}}-t_{j}^{\Xi}\right| & \leq C \int_{t}^{t+\min \left\{t_{j}^{\hat{\bar{\Xi}}}, t_{j}^{\Xi}\right\}}\left(\left|x_{j}^{\hat{\Xi}}(s, t, 1)-x_{j}^{\Xi}(s, t, 1)\right|+\|\hat{\Xi}-\Xi\|_{C^{0}([0,+\infty) \times[0,1])}\right) \mathrm{d} s \\
& \leq C\|\hat{\Xi}-\Xi\|_{C^{0}([0,+\infty) \times[0,1]) .}
\end{aligned}
$$

Combining (2.38) and (2.39) yields (2.37). One can also verify (2.25) by direct/similar computations and by using the fact

$$
\left|x_{j}^{\Xi}\left(t^{\prime}, s^{\prime}, \xi^{\prime}\right)-x_{j}^{\Xi}(t, s, \xi)\right| \leq C\left(\left|t^{\prime}-t\right|+\left|s^{\prime}-s\right|+\left|\xi^{\prime}-\xi\right|\right) .
$$

We now give the

Proof of Lemma 2.3. In what follows, for notational ease, we ignore the dependence of $H$ on $w_{0}$ and denote $H(t, \Xi, \varphi(t, \cdot))$ instead of $H\left(t, \Xi, \varphi(t, \cdot), w_{0}\right)$. Fix an appropriate $w^{(0)}$ such that $\left(w^{(0)}, w_{0}\right) \in \mathcal{D}_{\tau}$ and $\left\|w^{(0)}\right\|_{C^{1}([0,+\infty) \times[0,1])} \leq C\left\|w_{0}\right\|_{C^{1}([0,1])}$; we thus assumed implicitly here that $\left\|w_{0}\right\|_{C^{1}([0,1])}$ is sufficiently small. For $l \geq 0$, let $w^{(l+1)}$ be the unique $C^{1}$-solution of

$$
\left\{\begin{array}{cl}
\partial_{t} w^{(l+1)}(t, x)=\Sigma\left(x, w^{(l)}(t, x)\right) \partial_{x} w^{(l+1)}(t, x)+f\left(t, x, w^{(l+1)}(t, x)\right) & \text { in }[0,+\infty) \times[0,1], \\
w_{-}^{(l+1)}(t, 0)=\mathrm{B}\left(w_{+}^{(l+1)}(t, 0)\right) & \text { for } t \in[0,+\infty), \\
w_{+}^{(l+1)}(t, 1)=H\left(t, w^{(l)}(t+\cdot, \cdot), w^{(l+1)}(t, \cdot)\right) & \text { for } t \in[0,+\infty), \\
w^{(l+1)}(0, \cdot)=w_{0}(\cdot) & \text { in }[0,1],
\end{array}\right.
$$

and set

$$
W^{(l)}(t, x)=\partial_{t} w^{(l)}(t, x) \text { for }(t, x) \in[0,+\infty) \times[0,1] .
$$

The existence and uniqueness of $w^{(l+1)}$ follows from Lemma 2.1. Indeed, the compatibility conditions at $x=0$ follow from the fact $w^{(l)}(0, \cdot)=w_{0}(\cdot)$ and the compatibility conditions at $x=1$ follow from the assumption on 
$H$ for the existence of $C^{1}$-solutions of the system (2.28). We have

$$
\left\{\begin{aligned}
\partial_{t} W^{(l+1)}(t, x)= & \Sigma\left(x, w^{(l)}(t, x)\right) \partial_{x} W^{(l+1)}(t, x) \\
& +f_{1}(t, x) W^{(l+1)}(t, x)+f_{2}(t, x) \text { for }(t, x) \in[0,+\infty) \times[0,1], \\
W_{-}^{(l+1)}(t, 0)= & \nabla \mathrm{B}\left(w^{(l+1)}(t, 0)\right) W_{+}^{(l+1)}(t, 0) \text { for } t \in[0,+\infty), \\
W_{+}^{(l+1)}(t, 1)= & \partial_{t} H\left(t, w^{(l)}(t+\cdot, \cdot), w^{(l+1)}(t, \cdot)\right)+\left\langle\partial_{\Xi} H\left(t, w^{(l)}(t+\cdot, \cdot), w^{(l+1)}(t, \cdot)\right), W^{(l)}(t+\cdot, \cdot)\right\rangle \\
& +\left\langle\partial_{\varphi} H\left(t, w^{(l)}(t+\cdot, \cdot), w^{(l+1)}(t, \cdot)\right), W^{(l+1)}(t, \cdot)\right\rangle \text { for } t \in[0,+\infty), \\
W^{(l+1)}(0, \cdot)= & \Sigma\left(\cdot, w_{0}(x)\right) w_{0}^{\prime}(\cdot)+f\left(0, x, w_{0}(x)\right) \text { in }[0,1],
\end{aligned}\right.
$$

where

$$
f_{1}(t, x)=\partial_{y} \Sigma\left(x, w^{(l)}(t, x)\right) W^{(l)}(t, x) \Sigma^{-1}\left(x, w^{(l)}(t, x)\right)+\partial_{y} f\left(t, x, w^{(l+1)}(t, x)\right),
$$

and

$$
f_{2}(t, x)=\partial_{t} f\left(t, x, w^{(l+1)}(t, x)\right)-\partial_{y} \Sigma\left(x, w^{(l)}(t, x)\right) W^{(l)}(t, x) \Sigma^{-1}\left(x, w^{(l)}(t, x)\right) f\left(t, x, w^{(l+1)}(t, x)\right) .
$$

We have, since $H_{2}\left(t, w^{(l)}(t+\cdot), 0\right)=0$ by $(2.20)$,

$$
\begin{aligned}
\left|\partial_{t} H\left(t, w^{(l)}(t+\cdot, \cdot), w^{(l+1)}(t, \cdot)\right)\right| \stackrel{(2.18),(2.21)}{\leq} & C\left(\left|\partial_{t} H_{1}(t)\right|+\left\|w^{(l)}\right\|_{C^{0}([0,+\infty) \times[0,1])} \| w^{(l+1)}(t, \cdot)\right) \|_{C^{1}([0,1])} \\
& +\|\left(w^{(l+1)}(t, \cdot)\left\|_{C^{0}([0, a])}+\right\|\left(w^{(l+1)}(t, \cdot) \|_{L^{p}([0,1])}\right)\right.
\end{aligned}
$$

and

$$
\begin{aligned}
& \left|\left\langle\partial_{\varphi} H\left(t, w^{(l)}(t+\cdot, \cdot), w^{(l+1)}(t, \cdot)\right), W^{(l+1)}(t, \cdot)\right\rangle\right| \\
& \quad \stackrel{(2.22)}{\leq} C\left(\left\|W^{(l+1)}(t, \cdot)\right\|_{C^{0}([0, a])}+\left\|W^{(l+1)}(t, \cdot)\right\|_{L^{p}(0,1)}\right) .
\end{aligned}
$$

By introducing $\|\cdot\|_{0}$ and $\|\cdot\|_{1}$ as in (2.10) and (2.11), and using the above three inequalities, one can prove that

$$
\left\|w^{(l+1)}\right\|_{C^{1}([0,+\infty) \times[0,1])} \leq C\left(\sup _{t>0}\left(\left|H_{1}(t)\right|+\left|\partial_{t} H_{1}(t)\right|\right)+\left\|w_{0}\right\|_{C^{1}([0,1])}\right)
$$

if $\left\|w^{(l)}\right\|_{C^{1}([0,+\infty) \times[0,1])} \leq \varepsilon$ and $\varepsilon$ is sufficiently small. The smallness of $\varepsilon$ is also used to absorb the second term of the RHS of (2.42) and the RHS of (2.43). It follows from (2.19) that there exists a constant $0<C_{3}(\varepsilon)<\varepsilon$, 
independent of $w_{0}$ such that

$$
\left\|w^{(l)}\right\|_{C^{1}([0,+\infty) \times[0,1])} \leq C \varepsilon
$$

if

$$
\left\|w_{0}\right\|_{C^{1}[0,1]} \leq C_{3}(\varepsilon) \text { and } \varepsilon \text { is sufficiently small. }
$$

This fact will be assumed from now on.

Set, for $l \geq 1$,

$$
V^{(l)}=w^{(l)}-w^{(l-1)} \text { in }[0,+\infty) \times[0,1] .
$$

We have

$$
\left\{\begin{aligned}
\partial_{t} V^{(l+1)}(t, x)= & \Sigma\left(x, w^{(l)}(t, x)\right) \partial_{x} V^{(l+1)}(t, x) \\
& +\left(\Sigma\left(x, w^{(l)}(t, x)\right)-\Sigma\left(x, w^{(l-1)}(t, x)\right)\right) \partial_{x} w^{(l)}(t, x) \\
& +f\left(t, x, w^{(l+1)}(t, x)\right)-f\left(t, x, w^{(l)}(t, x)\right) \text { in }[0,+\infty) \times[0,1], \\
V_{-}^{(l+1)}(t, 0)= & \mathrm{B}\left(w_{+}^{(l+1)}(t, 0)\right)-\mathrm{B}\left(w_{+}^{(l)}(t, 0)\right) \text { for } t \in[0,+\infty), \\
V_{+}^{(l+1)}(t, 1)= & H\left(t, w^{(l)}(t+\cdot, \cdot), w^{(l+1)}(t, \cdot)\right)-H\left(t, w^{(l-1)}(t+\cdot, \cdot), w^{(l)}(t, \cdot)\right) \text { for } t \in[0,+\infty), \\
V^{(l+1)}(0, \cdot)= & 0 \text { in }[0,1] .
\end{aligned}\right.
$$

Note that, by $(2.45)$,

$$
\begin{gathered}
\left|\left(\Sigma\left(x, w^{(l)}(t, x)\right)-\Sigma\left(x, w^{(l-1)}(t, x)\right)\right) \partial_{x} w^{(l-1)}(t, x)\right| \stackrel{\Sigma \in C^{1}}{\leq} C \varepsilon\left|V^{(l)}(t, x)\right|, \\
\left|f\left(t, x, w^{(l+1)}(t, x)\right)-f\left(t, x, w^{(l)}(t, x)\right)\right| \stackrel{f \in C^{1}}{\leq} C\left|V^{(l+1)}(t, x)\right|, \\
\left|\mathrm{B}\left(w_{+}^{(l+1)}(t, 0)\right)-\mathrm{B}\left(w_{+}^{(l)}(t, 0)\right)\right| \stackrel{\mathrm{B} \in C^{1}}{\leq} C\left|V_{+}^{(l+1)}(t, 0)\right|, \\
\left|H\left(t, w^{(l)}(t+\cdot, \cdot), w^{(l+1)}(t, \cdot)\right)-H\left(t, w^{(l-1)}(t+\cdot, \cdot), w^{(l)}(t, \cdot)\right)\right| \\
\stackrel{(2.21)}{\leq} C\left(\varepsilon\left\|V^{(l)}(t+\cdot, \cdot)\right\|_{C^{0}([0,+\infty] \times[0,1])}+\left\|V^{(l+1)}(t, \cdot)\right\|_{C^{0}([0, a])}+\left\|V^{(l+1)}(t, \cdot)\right\|_{L^{p}([0,1])}\right) .
\end{gathered}
$$

Set

$$
Y_{l}(t)=\max _{1 \leq i \leq n} \max _{(s, x) \in[0, t] \times[0,1]}\left|\mathrm{e}^{-L_{1} s-L_{2} x} V_{i}^{(l)}(s, x)\right| .
$$

It follows that, provided that $L_{2}$ is large and $L_{1}$ is much larger than $L_{2}$,

$$
Y_{l+1}(t) \leq \int_{0}^{t}\left(\alpha Y_{l+1}(s)+\beta Y_{l}(s)\right) \mathrm{d} s+C \varepsilon Y_{l}(T),
$$


for some $\alpha, \beta>0$. By multiplying the above inequality with $\mathrm{e}^{-L t}$ for some large positive constant $L$, one can derive that, for $\varepsilon$ sufficiently small,

$$
\max _{t \in[0, T]} Y_{l+1}(t) \mathrm{e}^{-L t} \leq \frac{1}{2} \max _{t \in[0, T]} Y_{l}(t) \mathrm{e}^{-L t} .
$$

This implies

$$
w^{(l)} \text { converges in } C^{0}([0,+\infty) \times[0,1]) \text {. }
$$

Set

$$
\rho\left(\eta, w^{(l)}\right)=\sup _{t, x} \mathrm{e}^{-L_{1} t-L_{2} x} \sup _{\substack{t^{\prime}, x^{\prime} \\\left|(t, x)-\left(t^{\prime}, x^{\prime}\right)\right| \leq \eta}}\left|\left(\partial_{t}\left(w^{(l)}\left(t^{\prime}, x^{\prime}\right)-w^{(l)}(t, x)\right), \partial_{x}\left(w^{(l)}\left(t^{\prime}, x^{\prime}\right)-w^{(l)}(t, x)\right)\right)\right|
$$

and

$$
\rho\left(\eta, w_{0}\right)=\sup _{\left|x-x^{\prime}\right| \leq \eta}\left|w_{0}^{\prime}\left(x^{\prime}\right)-w_{0}^{\prime}(x)\right| .
$$

Define the flows

$$
\frac{\mathrm{d}}{\mathrm{d} t} x_{j}^{(l)}(t, s, \xi)=\lambda_{j}\left(x_{j}^{(l)}(t, s, \xi), w^{(l)}\left(t, x_{j}^{(l)}(t, s, \xi)\right)\right) \quad \text { and } \quad x_{j}^{(l)}(s, s, \xi)=\xi \text { for } 1 \leq j \leq k,
$$

and

$$
\frac{\mathrm{d}}{\mathrm{d} t} x_{j}^{(l)}(t, s, \xi)=-\lambda_{j}\left(x_{j}^{(l)}(t, s, \xi), w^{(l)}\left(t, x_{j}^{(l)}(t, s, \xi)\right)\right) \quad \text { and } \quad x_{j}^{(l)}(s, s, \xi)=\xi \text { for } k+1 \leq j \leq k+m .
$$

By (1.3) and the fact $\left\|w^{(l)}\right\|_{C^{1}([0,+\infty) \times[0,1])} \leq C \varepsilon$, one has, for $t, t^{\prime}<2 T$,

$$
\left|x_{j}^{(l)}\left(t^{\prime}, s^{\prime}, \xi^{\prime}\right)-x_{j}^{(l)}(t, s, \xi)\right| \leq C_{T}\left(\left|t^{\prime}-t\right|+\left|s-s^{\prime}\right|+\left|\xi^{\prime}-\xi\right|\right) .
$$

Recall, in the definition of $\rho_{2}$, that $a \in[0,1)$ and $1 \leq p<+\infty$. Use (2.24), (2.25) and (2.47), and consider (2.41). As in the proof of Lemma 3.2 from [4] (also the proof of Lem. 2.1), one can prove that

$$
\rho\left(\eta, w^{(l)}\right) \leq C \rho\left(C \eta, w_{0}\right)+C \eta+C \rho_{1}(C \eta)
$$

(see also the arguments in [9], Appendix B and [14] on pp. 58-59, 62-64, 88-90).

Combining (2.46) and (2.48), and applying the Ascoli theorem, one derives that

$$
w^{(l)} \text { converges in }\left(C^{1}([0,+\infty) \times[0,1])\right)^{n} .
$$

It is clear that the limit is a $C^{1}$-solution of (1.1), (1.5), and (2.29).

We next establish the uniqueness. Assume that $w$ and $\hat{w}$ are two $C^{1}$-solutions of (1.1), (1.5), and (2.29). Set $u=\hat{w}-w$ in $[0,+\infty) \times[0,1]$. Then

$$
\partial_{t} u(t, x)=A(t, x) \partial_{x} u(t, x)+\tilde{f}(t, x, u(t, x)),
$$


where

$$
\begin{gathered}
A(t, x)=\Sigma(x, w(t, x)), \\
\widetilde{f}(t, x, u(t, x))=(\Sigma(x, w(t, x)+u(t, x))-\Sigma(x, w(t, x))) \partial_{x} \hat{w}(t, x) \\
+f(t, x, w(t, x)+u(t, x))-f(t, x, w(t, x)) .
\end{gathered}
$$

Moreover,

$$
\begin{gathered}
u_{-}(t, 0)=g\left(t, u_{+}(t, 0)\right):=\mathrm{B}\left(w_{+}(t, 0)+u_{+}(t, 0)\right)-\mathrm{B}\left(w_{+}(t, 0)\right), \\
u_{+}(t, 0)=h(t, u(t+\cdot, \cdot)):=H(t, w+u, w+u)-H(t, w, w),
\end{gathered}
$$

and

$$
u(t=0, \cdot)=0 .
$$

Note that

$$
\begin{aligned}
& |\widetilde{f}(t, x, u(t, x))| \leq C|u(t, x)|, \\
& \left|g\left(t, u_{+}(t, 0)\right)\right| \leq C\left|u_{+}(t, 0)\right|,
\end{aligned}
$$

and

$$
|h(t, u(t+\cdot, \cdot))| \stackrel{(2.21)}{\leq} C\left(\varepsilon\|u\|_{C^{0}([0,+\infty) \times[0,1])}+\|u(t, \cdot)\|_{C^{0}([0, a])}+\|u(t, \cdot)\|_{L^{p}([0,1])}\right) .
$$

Let $U \in(C([0,+\infty) \times[0,1]))^{n}$, with $U(t, \cdot)=0$ for $t>T$, be a solution of the system

$$
\left\{\begin{array}{cl}
\partial_{t} U(t, x)-A(t, x) \partial_{x} U(t, x)=\tilde{f}(t, x, u(t, x)) & \text { in }[0,+\infty) \times[0,1], \\
U_{-}(t, 0)=g\left(t, U_{+}(t, 0)\right) & \text { for } t \in[0,+\infty), \\
U_{+}(t, 0)=h(t, u(t+\cdot, \cdot)) & \text { for } t \in[0,+\infty), \\
U(t=0, \cdot)=0 & \text { in }[0,1],
\end{array}\right.
$$

and set

$$
Y(t)=\max _{1 \leq i \leq n} \max _{(s, x) \in[0, t] \times[0,1]}\left|\mathrm{e}^{-L_{1} s-L_{2} x} U_{i}(s, x)\right|
$$

and

$$
Z(t)=\max _{1 \leq i \leq n} \max _{(s, x) \in[0, t] \times[0,1]}\left|\mathrm{e}^{-L_{1} s-L_{2} x} u_{i}(s, x)\right| .
$$


As in the proof of Lemma 3.2 from [4], one can prove that, if $L_{2}$ is large and $L_{1}$ is much larger than $L_{2}$,

$$
Y(t) \leq C \int_{0}^{t}(Y(s)+Z(s)) \mathrm{d} s+C \varepsilon Z(T)
$$

By multiplying the above inequality with $\mathrm{e}^{-L t}$, for some large positive constant $L$, one has

$$
\max _{t \in[0, T]} Y(t) \mathrm{e}^{-L t} \leq \frac{1}{2} \max _{t \in[0, T]} Z(t) \mathrm{e}^{-L t} .
$$

if $\varepsilon$ is sufficiently small. As a consequence, by taking $U=u$, one has, for $\varepsilon$ sufficiently small,

$$
u=0
$$

and the uniqueness follows. The proof is complete.

Remark 2.4. The proof of Lemma 2.3 is inspired from [4] using the approach for quasilinear hyperbolic equations in [14], Chapter 1 and [3], Chapter 3.

\subsection{Proof of Theorem 1.1}

We consider two cases $m>k$ and $m \leq k$ separately.

Case 1: $m>k$. Consider the last equation of (1.5). Impose the condition $w_{k}(t, 0)=0$. Using (1.10) with $i=1$ and the implicit function theorem, one can then write the last equation of (1.5) under the form

$$
w_{m+k}(t, 0)=M_{k}\left(w_{k+1}(t, 0), \ldots, w_{m+k-1}(t, 0)\right)
$$

for some $C^{2}$ nonlinear map $M_{k}$ from $U_{k}$ into $\mathbb{R}$ for some neighborhood $U_{k}$ of $0 \in \mathbb{R}^{m-1}$ with $M_{k}(0)=0$ provided that $\left|w_{+}(t, 0)\right|$ is sufficiently small.

Consider the last two equations of (1.5) and impose the condition $w_{k}(t, 0)=w_{k-1}(t, 0)=0$. Using (1.10) with $i=2$ and the Gaussian elimination approach, one can then write these two equations under the form (2.49) and

$$
w_{m+k-1}(t, 0)=M_{k-1}\left(w_{k+1}(t, 0), \ldots, w_{m+k-2}(t, 0)\right),
$$

for some $C^{2}$ nonlinear map $M_{k-1}$ from $U_{k-1}$ into $\mathbb{R}$ for some neighborhood $U_{k-1}$ of $0 \in \mathbb{R}^{m-2}$ with $M_{k-1}(0)=0$ provided that $\left|w_{+}(t, 0)\right|$ is sufficiently small, etc. Finally, consider the $k$ equations of (1.5) and impose the condition $w_{k}(t, 0)=\cdots=w_{1}(t, 0)=0$. Using (1.10) with $i=k$ and the Gaussian elimination approach, one can then write these $k$ equations under the form $(2.49),(2.50), \ldots$, and

$$
w_{m+1}(t, 0)=M_{1}\left(w_{k+1}(t, 0), \ldots, w_{m}(t, 0)\right)
$$

for some $C^{2}$ nonlinear map $M_{1}$ from $U_{1}$ into $\mathbb{R}$ for some neighborhood $U_{1}$ of $0 \in \mathbb{R}^{m-k}$ with $M_{1}(0)=0$ provided that $\left|w_{+}(t, 0)\right|$ is sufficiently small. These nonlinear maps $M_{1}, \ldots, M_{k}$ will be used in the construction of feedbacks.

We next introduce the flows along the characteristic curves. Set

$$
\frac{\mathrm{d}}{\mathrm{d} t} x_{j}(t, s, \xi)=\lambda_{j}\left(x_{j}(t, s, \xi), w\left(t, x_{j}(t, s, \xi)\right)\right) \quad \text { and } \quad x_{j}(s, s, \xi)=\xi \text { for } 1 \leq j \leq k
$$


and

$$
\frac{\mathrm{d}}{\mathrm{d} t} x_{j}(t, s, \xi)=-\lambda_{j}\left(x_{j}(t, s, \xi), w\left(t, x_{j}(t, s, \xi)\right)\right) \quad \text { and } \quad x_{j}(s, s, \xi)=\xi \text { for } k+1 \leq j \leq k+m
$$

We do not precise at this stage the domain of the definition of $x_{j}$. Later, we only consider the flows in the regions where the solution $w$ is well-defined.

To arrange the compatibility of our controls, we introduce auxiliary variables satisfying autonomous dynamics, which will be defined later. Set $\delta=T-T_{\text {opt }}>0$. For $t \geq 0$, define, for $k+1 \leq j \leq k+m$,

$$
\zeta_{j}(0)=w_{0, j}(1), \quad \zeta_{j}^{\prime}(0)=\lambda_{j}\left(0, w_{0}(1)\right) w_{0, j}^{\prime}(1), \quad \zeta_{j}(t)=0 \text { for } t \geq \delta / 2
$$

and

$$
\eta_{j}(0)=1, \quad \eta_{j}^{\prime}(0)=0, \quad \eta_{j}(t)=0 \text { for } t \geq \delta / 2
$$

We will construct the dynamics for $\zeta_{j}$ and $\eta_{j}$ at the end of the proof of Theorem 1.1.

We are ready to construct a feedback law leading to finite-time stabilization in the time $T$. Let $t_{m+k}$ be such that

$$
x_{m+k}\left(t+t_{m+k}, t, 1\right)=0 .
$$

It is clear that $t_{m+k}$ depends only on the current state $w(t, \cdot)$. Let $D_{m+k}=D_{m+k}(t) \subset \mathbb{R}^{2}$ be the open set whose boundary is $\{t\} \times[0,1],\left[t, t+t_{m+k}\right] \times\{0\}$, and $\left\{\left(s, x_{m+k}(s, t, 1)\right) ; s \in\left[t, t+t_{m+k}\right]\right\}$. Then $D_{m+k}$ depends only on the current state as well. This implies

$$
x_{k+1}\left(t, t+t_{m+k}, 0\right), \ldots, x_{k+m-1}\left(t, t+t_{m+k}, 0\right) \text { are well-defined by the current state } w(t, \cdot) .
$$

As a consequence, the feedback

$$
\begin{aligned}
& w_{m+k}(t, 1)=\zeta_{m+k}(t) \\
& \quad+\left(1-\eta_{m+k}(t)\right) M_{k}\left(w_{k+1}\left(t, x_{k+1}\left(t, t+t_{m+k}, 0\right)\right), \ldots, w_{k+m-1}\left(t, x_{k+m-1}\left(t, t+t_{m+k}, 0\right)\right)\right)
\end{aligned}
$$

is well-defined by the current state $w(t, \cdot)$.

We then consider the system (1.1), (1.5), and the feedback (2.54). Let $t_{m+k-1}$ be such that

$$
x_{m+k-1}\left(t+t_{m+k-1}, t, 1\right)=0 .
$$

It is clear that $t_{m+k-1}$ depends only on the current state $w(t, \cdot)$ and the feedback law (2.54). Let $D_{m+k-1}=D_{m+k-1}(t) \subset \mathbb{R}^{2}$ be the open set whose boundary is $\{t\} \times[0,1],\left[t, t+t_{m+k-1}\right] \times\{0\}$, and $\left\{\left(s, x_{m+k-1}(s, t, 1)\right) ; s \in\left[t, t+t_{m+k-1}\right]\right\}$. Then $D_{m+k-1}$ depends only on the current state. This implies

$$
x_{k+1}\left(t, t+t_{m+k-1}, 0\right), \ldots, x_{k+m-2}\left(t, t+t_{m+k-1}, 0\right) \text { are well-defined by the current state } w(t, \cdot) .
$$

As a consequence, the feedback

$$
w_{m+k-1}(t, 1)=\zeta_{m+k-1}(t)
$$




$$
+\left(1-\eta_{m+k-1}(t)\right) M_{k-1}\left(w_{k+1}\left(t, x_{k+1}\left(t, t+t_{m+k-1}, 0\right)\right), \ldots, w_{k+m-2}\left(t, x_{k+m-2}\left(t, t+t_{m+k-1}, 0\right)\right)\right)
$$

is well-defined by the current state $w(t, \cdot)$.

We continue this process and finally reach the system $(1.1),(1.5),(2.54), \ldots$

$$
\begin{aligned}
w_{m+2}(t, 1)= & \zeta_{m+2}(t) \\
& +\left(1-\eta_{m+2}(t)\right) M_{2}\left(w_{k+1}\left(t, x_{k+1}\left(t, t+t_{m+2}, 0\right)\right), \ldots, w_{m+1}\left(t, x_{m+1}\left(t, t+t_{m+2}, 0\right)\right)\right) .
\end{aligned}
$$

Let $t_{m+1}$ be such that

$$
x_{m+1}\left(t+t_{m+1}, t, 1\right)=0 .
$$

It is clear that $t_{m+1}$ depends only on the current state $w(t, \cdot)$ and the feedback law $(2.54)-(2.56)$. Let $D_{m+1}=$ $D_{m+1}(t) \subset \mathbb{R}^{2}$ be the open set whose boundary is $\{t\} \times[0,1],\left[t, t+t_{m+1}\right] \times\{0\}$, and $\left\{\left(s, x_{m+1}(s, t, 1)\right) ; s \in\right.$ $\left.\left[t, t+t_{m+1}\right]\right\}$. Then $D_{m+1}$ depends only on the current state. This implies

$$
x_{k+1}\left(t, t+t_{m+1}, 0\right), \ldots, x_{m}\left(t, t+t_{m+1}, 0\right) \text { are well-defined by the current state } w(t, \cdot) \text {. }
$$

As a consequence, the feedback

$$
\begin{aligned}
w_{m+1}(t, 1)= & \zeta_{m+1}(t) \\
& +\left(1-\eta_{m+1}(t)\right) M_{1}\left(w_{k+1}\left(t, x_{k+1}\left(t, t+t_{m+1}, 0\right)\right), \ldots, w_{m}\left(t, x_{m}\left(t, t+t_{m+1}, 0\right)\right)\right)
\end{aligned}
$$

is well-defined by the current state $w(t, \cdot)$.

To complete the feedback for the system, we consider, for $k+1 \leq j \leq m$,

$$
w_{j}(t, 1)=\zeta_{j}(t)
$$

We will establish that the feedback constructed gives the finite-time stabilization in the time $T$ if $\varepsilon$ is sufficiently small. To this end, we first claim that

$$
\text { the system }(1.1),(1.5),(2.54)-(2.57) \text { is well-posed if } \varepsilon \text { is sufficiently small. }
$$

Indeed, it is clear to see that the feedback is given by

$$
H\left(t, w(t+\cdot), w(t, \cdot), w_{0}\right),
$$

where $H$ is given by (2.33)-(2.36). The well-posedness for the feedback law is now a consequence of Lemma 2.3 through the example mentioned and examined right after it.

From (2.31) and (2.32), we have, for $t \geq \delta / 2$,

$$
\zeta_{j}(t)=0 \text { for } k+1 \leq j \leq k+m
$$


It follows that, for $t \geq \delta / 2$, the feedback law (2.54)-(2.57) has the form

$$
\begin{gathered}
w_{m+k}(t, 1)=M_{k}\left(w_{k+1}\left(t, x_{k+1}\left(t, t+t_{m+k}, 0\right)\right), \ldots, w_{k+m-1}\left(t, x_{k+m-1}\left(t, t+t_{m+k}, 0\right)\right)\right), \\
w_{m+k-1}(t, 1)=M_{k-1}\left(w_{k+1}\left(t, x_{k+1}\left(t, t+t_{m+k-1}, 0\right)\right), \ldots, w_{k+m-2}\left(t, x_{k+m-2}\left(t, t+t_{m+k-1}, 0\right)\right)\right), \\
w_{m+1}(t, 1)=M_{1}\left(w_{k+1}\left(t, x_{k+1}\left(t, t+t_{m+1}, 0\right)\right), \ldots, w_{m}\left(t, x_{m}\left(t, t+t_{m+1}, 0\right)\right)\right) .
\end{gathered}
$$

Set

$$
\hat{t}=\max \left\{\hat{t}_{k+1}, \ldots, \hat{t}_{k+m}\right\}
$$

where $\hat{t}_{j}$, for $k+1 \leq j \leq k+m$, is defined by

$$
x_{j}\left(\hat{t}_{j}+\delta / 2, \delta / 2,1\right)=0
$$

It follows from the characteristic method that

$$
w_{j}(t, \cdot)=0 \text { for } t \geq \hat{t}+\delta / 2 \text { for } k+1 \leq j \leq m,
$$

then for $j=m+1$, then for $j=m+2, \ldots$, then for $j=m+k$.

Using the characteristic method again, we have, by the choice of $M_{k}$,

$$
w_{k}(t, 0)=0 \text { for } t \geq \delta / 2+\hat{t}_{m+k},
$$

by the choice of $M_{k}$ and $M_{k-1}$,

$$
w_{k-1}(t, 0)=0 \text { for } t \geq \delta / 2+\hat{t}_{m+k-1},
$$

$\ldots$, and, by the choice of $M_{k}, M_{k-1}, \ldots, M_{1}$,

$$
w_{1}(t, 0)=0 \text { for } t \geq \delta / 2+\hat{t}_{m+1} .
$$

Let $\hat{t}_{k}, \ldots, \hat{t}_{1}$ be such that

$$
\begin{aligned}
& x_{k}\left(\hat{t}_{k}+\delta / 2+\hat{t}_{m+k}, \delta / 2+\hat{t}_{m+k}, 0\right)=1, \\
& x_{1}\left(\hat{t}_{1}+\delta / 2+\hat{t}_{m+1}, \delta / 2+\hat{t}_{m+1}, 0\right)=1 .
\end{aligned}
$$

Using the characteristic method, we derive that

$$
w_{k}(t, \cdot)=0 \text { for } t \geq \delta / 2+\hat{t}_{m+k}+\hat{t}_{k},
$$




$$
w_{1}(t, \cdot)=0 \text { for } t \geq \delta / 2+\hat{t}_{m+1}+\hat{t}_{1} .
$$

The conclusion follows by noting that

$$
\left|\hat{t}_{j}-\tau_{j}\right| \leq \delta / 4 \text { for } 1 \leq j \leq k+m,
$$

if $\varepsilon$ is sufficiently small thanks to (2.19) and (2.30).

Case 2: $m \leq k$. We consider the following feedback law

$$
\begin{aligned}
w_{m+k}(t, 1)= & \zeta_{m+k}(t) \\
& +\left(1-\eta_{m+k}(t)\right) M_{k}\left(w_{k+1}\left(t, x_{k+1}\left(t, t+t_{m+k}, 0\right)\right), \ldots, w_{k+m-1}\left(t, x_{k+m-1}\left(t, t+t_{m+k}, 0\right)\right)\right),
\end{aligned}
$$

$\cdots$

$$
w_{k+2}(t, 1)=\zeta_{k+2}(t)+\left(1-\eta_{k+2}(t)\right) M_{2}\left(w_{k+1}\left(t, x_{k+1}\left(t, t+t_{k+2}, 0\right)\right)\right),
$$

and

$$
w_{k+1}(t, 1)=\zeta_{k+1}(t)
$$

The conclusion now follows by the same arguments. The details are omitted.

It remains to construct a dynamics for $\zeta_{j}$ and $\eta_{j}$. To this end, inspired by $[7,15]$, we write $\zeta_{j}=\varphi_{j}+\psi_{j}$ where $\varphi_{j}$ and $\psi_{j}$ satisfy the dynamics

$$
\varphi_{j}^{\prime}(t)=-\frac{\alpha \varphi_{j}}{\left(\varphi_{j}^{2}+\psi_{j}^{2}\right)^{1 / 3}} \quad \text { and } \quad \psi_{j}^{\prime}(t)=-\frac{\beta \varphi_{j}}{\left(\varphi_{j}^{2}+\psi_{j}^{2}\right)^{1 / 3}}
$$

with $Y=\left(\varphi_{j}(0)^{2}+\psi_{j}(0)^{2}\right)^{1 / 3}$

$$
\varphi_{j}(0)+\psi_{j}(0)=a, \quad-\alpha \varphi_{j}(0)-\beta \psi_{j}(0)=b Y,
$$

where $a=w_{0, j}(0)$ and $b=\lambda_{j}\left(0, w_{0}(1)\right) w_{0, j}^{\prime}(1)$. Here $\alpha$ and $\beta$ are two distinct real numbers. We now show that under appropriate choice of $\alpha$ and $\beta, \varphi_{j}(0)$ and $\psi_{j}(0)$ can be chosen as continuous functions of $a$ and $b$ for $|(a, b)|$ sufficiently small. Indeed, consider the equation $P_{a, b}(Y)=0$, where

$$
P_{a, b}(Y):=(\alpha-\beta)^{2} Y^{3}-\left(2 b^{2} Y^{2}+2 a b(\alpha+\beta) Y+a^{2}\left(\alpha^{2}+\beta^{2}\right)\right) .
$$

One has, for $Y>0$ and $P_{a, b}(Y)=0$,

$$
Y P_{a, b}^{\prime}(Y)=2 b^{2} Y^{2}+4 a b(\alpha+\beta) Y+3\left(\alpha^{2}+\beta^{2}\right) a^{2} .
$$

In particular,

$$
P_{a, b}^{\prime}(Y)>0 \text { if } \alpha^{2}+\beta^{2}-4 \alpha \beta>0 \text { and if } a b \neq 0,
$$

and the equation $P_{a, b}(Y)=0$ has a unique positive solution in this case. In the case $a b=0$ and $a^{2}+b^{2}>0$, there is a unique positive solution of $P_{a, b}(Y)=0$ and in the case $a=b=0$, there is a unique solution $Y=0$. 
Fix $\alpha$ and $\beta$ such that $\alpha^{2}+\beta^{2}-4 \alpha \beta \neq 0$ and $\alpha \neq \beta$. Denote $\bar{Y}(a, b)$ the unique positive solution in the case $a^{2}+b^{2}>0$ and 0 for $(a, b)=(0,0)$. It suffices to prove that $\bar{Y}(a, b)$ is continuous with respect to $(a, b)$ for small $|(a, b)|$. Since $P_{a, b}(1)>0$ if $|(a, b)|$ is sufficiently small and $P_{a, b}(0)<0$ if $a \neq 0$, it follows that $\bar{Y}$ is bounded in a neighborhood $O$ of $(0,0)$. Since $P_{a, b}(Y)=0$ has a unique non-negative solution for $a \neq 0$, it follows that $\bar{Y}$ is continuous in $O \backslash\{(a, b) ; a=0\}$. Since $\alpha^{2}+\beta^{2}-4 \alpha \beta>0$, one has

$$
\frac{3}{2} b^{2} Y^{2}+2 a b(\alpha+\beta) Y+a^{2}\left(\alpha^{2}+\beta^{2}\right) \geq 0 .
$$

It follows that

$$
P_{a, b}(Y) \leq(\alpha-\beta)^{2} Y^{3}-\frac{1}{2} b^{2} Y^{2}
$$

This implies the continuity of $\bar{Y}$ on $O \cap\{(a, b) ; a=0$ and $b \neq 0\}$. The continuity of $\bar{Y}$ at $(0,0)$ is a consequence of the fact $P_{0,0}(Y)=0$ implies $Y=0$.

Similarly, one can build the dynamics for $\eta_{j}$. We now have $a=1$ and $b=0$. we write $\eta_{j}=\widetilde{\varphi}_{j}+\widetilde{\psi}_{j}$ where $\widetilde{\varphi}_{j}$ and $\widetilde{\psi}_{j}$ satisfy the dynamics

$$
\widetilde{\varphi}_{j}^{\prime}(t)=-\frac{\lambda^{5 / 3} \alpha \widetilde{\varphi}_{j}}{\left(\widetilde{\varphi}_{j}^{2}+\widetilde{\psi}_{j}^{2}\right)^{1 / 3}} \quad \text { and } \quad \widetilde{\psi}_{j}^{\prime}(t)=-\frac{\lambda^{5 / 3} \beta \widetilde{\varphi}_{j}}{\left(\widetilde{\varphi}_{j}^{2}+\widetilde{\psi}_{j}^{2}\right)^{1 / 3}}
$$

where $\lambda$ is a large, positive constant defined later. One can check that $\widetilde{\varphi}_{j}(t)=\lambda \varphi(\lambda t)$ and $\widetilde{\psi}_{j}(t)=\lambda \psi(\lambda t)$ where $\varphi_{j}$ and $\psi_{j}$ are solutions of (2.70) and

$$
\varphi_{j}(0)+\psi_{j}(0)=\lambda^{-1} a, \quad-\alpha \varphi_{j}(0)-\beta \psi_{j}(0)=0,
$$

instead of (2.71). One then can obtain the dynamics for $\eta_{j}$ by choosing $\lambda$ large enough.

Acknowledgements. The authors were partially supported by ANR Finite4SoS ANR-15-CE23-0007. H.-M. Nguyen thanks Fondation des Sciences Mathématiques de Paris (FSMP) for the Chaire d'excellence which allows him to visit Laboratoire Jacques Louis Lions and Mines ParisTech. This work has been done during this visit.

\section{REFERENCES}

[1] J. Auriol and F. Di Meglio, Minimum time control of heterodirectional linear coupled hyperbolic PDEs. Automatica J. IFAC 71 (2016) 300-307.

[2] G. Bastin and J.-M. Coron, Stability and boundary stabilization of 1-D hyperbolic systems, in Progress in Nonlinear Differential Equations and their Applications, Vol. 88. Birkhäuser/Springer, Cham (2016).

[3] A. Bressan, Hyperbolic systems of conservation laws, in Oxford Lecture Series in Mathematics and its Applications, Vol. 20. Oxford University Press, Oxford (2000).

[4] J.-M. Coron and H.-M. Nguyen, Optimal time for the controllability of linear hyperbolic systems in one-dimensional space. SIAM J. Control Optim. 57 (2019) 1127-1156.

[5] J.-M. Coron and H.-M. Nguyen, Null controllability of linear hyperbolic systems in one-dimensional space. Preprint arXiv: $1910.12268 \mathrm{v} 1(2019)$.

[6] J.-M. Coron and H.-M. Nguyen, Lyapunov functions and finite time stabilization in optimal time for homogeneous linear and quasilinear hyperbolic systems. Preprint arXiv: 2007.04104 (2020).

[7] J.-M. Coron, R. Vazquez, M. Krstic and G. Bastin, Local exponential $H^{2}$ stabilization of a $2 \times 2$ quasilinear hyperbolic system using backstepping. SIAM J. Control Optim. 51 (2013) 2005-2035.

[8] J.-M. Coron, L. Hu and G. Olive, Finite-time boundary stabilization of general linear hyperbolic balance laws via Fredholm backstepping transformation. Automatica J. IFAC $\mathbf{8 4}$ (2017) 95-100.

[9] L. Hu, Sharp time estimates for exact boundary controllability of quasilinear hyperbolic systems. SIAM J. Control Optim. 53 (2015) 3383-3410.

[10] L. Hu and G. Olive, Minimal time for the exact controllability of one-dimensional first-order linear hyperbolic systems by one-sided boundary controls. Preprint arXiv: 1901.06005 (2019). 
[11] M. Krstic and A. Smyshlyaev, Boundary control of PDEs, in Advances in Design and Control, Vol. 16. Society for Industrial and Applied Mathematics SIAM, Philadelphia, PA (2008).

[12] T.-T. Li, Controllability and observability for quasilinear hyperbolic systems. AIMS Series on Applied Mathematics, Vol. 3. American Institute of Mathematical Sciences AIMS, Springfield, MO; Higher Education Press, Beijing (2010).

[13] T.-T. Li and B. Rao, Local Exact Boundary Controllability for a Class Of Quasilinear Hyperbolic Systems, Vol. 23. (2002) 209-218.

[14] T.-T. Li and W.C. Yu, Boundary Value Problems for Quasilinear Hyperbolic Systems. Duke University Mathematics Series. V. Duke University, Mathematics Department. Durham, NC (1985).

[15] V. Perrollaz and L. Rosier, Finite-time stabilization of $2 \times 2$ hyperbolic systems on tree-shaped networks. SIAM J. Control Optim. 52 (2014) 143-163.

[16] D.L. Russell, Controllability and stabilizability theory for linear partial differential equations: recent progress and open questions. SIAM Rev. 20 (1978) 639-739.

[17] N. Weck, A remark on controllability for symmetric hyperbolic systems in one space dimension. SIAM J. Control Optim. 20 (1982) 1-8. 